\title{
Letter to the Editor concerning "Scoliosis convexity and organ anatomy are related" by T. P. C. Schlösser et al. (Eur Spine J; 2017: doi:10.1007/s00586-017-4970-5)
}

\author{
Zehao Jing $^{1} \cdot$ Feng Nan ${ }^{1}$
}

Received: 16 February 2017 / Accepted: 29 July 2017 / Published online: 11 October 2017

(c) Springer-Verlag GmbH Germany 2017

To the Editor,

We read with interest the above-mentioned article [Schlösser TPC, Semple T, Carr SB, et al. (2017) Scoliosis convexity and organ anatomy are related. Eur Spine J; doi:10.1007/ s00586-017-4970-5], which confirmed that there is a correlation between organ anatomy and curve convexity in idiopathic scoliosis (Discussion, line 7-9). We fear that this conclusion is questionable for the following reason: the increased prevalence of scoliosis ( $8 \%$ in this PCD population versus 1.5-3\% in the normal population) [Lonstein JE (1994) Adolescent idiopathic scoliosis. Lancet 344:1407-1412] and the potential link between cilia and scoliosis [Oliazadeh N, Franco A, Wang D, Moreau A (2015) Abnormalities in primary cilium of osteoblasts of adolescent idiopathic scoliosis patients. Cilia 4(Suppl 1):6; Grimes DT, Boswell
CW, Morante NF, Henkelman RM, Burdine RD, Ciruna B (2016) Zebrafish models of idiopathic scoliosis link cerebrospinal fluid flow defects to spine curvature. Science 352:1341-1344] both suggest that the scoliosis in this study is related to PCD. As we are not sure whether the scoliosis in this study is induced by PCD, it is more proper to name it as "PCD accompanied scoliosis" which should not be an idiopathic one. PCD-accompanied scoliosis may have different pathogenesis compared with idiopathic ones. So, it is confusing to generalize the conclusion in this article to all idiopathic scoliosis.

\section{Compliance with ethical standards}

Conflict of interest Zehao Jing and Feng Nan declare that they have no conflict of interest.
Feng Nan

nanf1974@163.com

1 Second Affiliated Hospital of Dalian Medical University, No. 467, Zhongshan Road, Shahekou District, Dalian, Liaoning Province, China 